\title{
FAIR DOMINATION NUMBER IN CACTUS GRAPHS
}

\author{
MAJID HAJIAN \\ Department of Mathematics \\ Shahrood University of Technology \\ Shahrood, Iran \\ AND \\ NADER JAFARI RAD \\ Department of Mathematics \\ Shahed University, Tehran, Iran \\ e-mail: n.jafarirad@gmail.com
}

\begin{abstract}
For $k \geq 1$, a $k$-fair dominating set (or just $k$ FD-set) in a graph $G$ is a dominating set $S$ such that $|N(v) \cap S|=k$ for every vertex $v \in V \backslash S$. The $k$-fair domination number of $G$, denoted by $f d_{k}(G)$, is the minimum cardinality of a $k \mathrm{FD}$-set. A fair dominating set, abbreviated FD-set, is a $k \mathrm{FD}$-set for some integer $k \geq 1$. The fair domination number, denoted by $f d(G)$, of $G$ that is not the empty graph, is the minimum cardinality of an FD-set in $G$. In this paper, aiming to provide a particular answer to a problem posed in [Y. Caro, A. Hansberg and M.A. Henning, Fair domination in graphs, Discrete Math. 312 (2012) 2905-2914], we present a new upper bound for the fair domination number of a cactus graph, and characterize all cactus graphs $G$ achieving equality in the upper bound of $f d_{1}(G)$.
\end{abstract}

Keywords: fair domination, cactus graph, unicyclic graph.

2010 Mathematics Subject Classification: 05C69.

\section{REFERENCES}

[1] Y. Caro, A. Hansberg and M.A. Henning, Fair domination in graphs, Discrete Math. 312 (2012) 2905-2914. doi:10.1016/j.disc.2012.05.006

[2] B. Chaluvaraju, M. Chellali and K.A. Vidya, Perfect $k$-domination in graphs, Australas. J. Combin. 48 (2010) 175-184. 
[3] B. Chaluvaraju and K.A. Vidya, Perfect dominating set graph of a graph $G$, Adv. Appl. Discrete Math. 2 (2008) 49-57.

[4] E.J. Cockayne, B.L. Hartnell, S.T. Hedetniemi and R. Laskar, Perfect domination in graphs, J. Comb. Inf. Syst. Sci. 18 (1993) 136-148.

[5] I.J. Dejter, Perfect domination in regular grid graphs, Australas. J. Combin. 42 (2008) 99-114.

[6] I.J. Dejter and A.A. Delgado, Perfect domination in rectangular grid graphs, J. Combin. Math. Combin. Comput. 70 (2009) 177-196.

[7] M.R. Fellows and M.N. Hoover, Perfect domination, Australas. J. Combin. 3 (1991) 141-150.

[8] M. Hajian and N. Jafari Rad, Trees and unicyclic graphs with large fair domination number, Util. Math. accepted.

[9] H. Hatami and P. Hatami, Perfect dominating sets in the Cartesian products of prime cycles, Electron. J. Combin. 14 (2007) \#N8.

[10] T.W. Haynes, S.T. Hedetniemi and P.J. Slater, Fundamentals of Domination in Graphs (Marcel Dekker Inc., New York, 1998).

Received 4 May 2017

Revised 5 September 2017

Accepted 19 September 2017 